\title{
Assessment of Biophysical Skin Properties at Different Body Sites in Hospitalized Old Patients: Results of a Pilot Study
}

\author{
M. Fiedler ${ }^{\mathrm{a}} \quad$ L.-C. Gerhardt ${ }^{\mathrm{b}} \quad$ S. Derler ${ }^{\mathrm{b}} \quad$ G. Bischofberger ${ }^{\mathrm{a}} \quad$ C. Hürny ${ }^{\mathrm{a}}$ \\ T. Münzer ${ }^{a, c}$ \\ ${ }^{a}$ Geriatrische Klinik, and ${ }^{b}$ Laboratory for Protection and Physiology, Empa, Swiss Federal Laboratories for Materials \\ Science and Technology, St. Gallen, and ${ }^{\complement}$ Geriatric University Hospital Bern, Bern, Switzerland
}

\section{Key Words}

Viscoelasticity $\cdot$ Skin aging $\cdot$ Decubitus risk

\begin{abstract}
Background: Skin aging is a risk factor for a decubitus and biophysical skin properties could help to identify persons at risk. Whether such biophysical properties of aged human skin differ between areas is undetermined. Objective: To investigate whether viscoelasticity, hydration or friction differ between important areas for decubitus risk. Methods: Pilot study in 32 (18 female, 14 male) acute and subacute old patients aged $81.9 \pm 5.9$ years $( \pm S D)$, without active skin disease after an average of 10 days of stay. Assessment of skin resilience/viscoelasticity $(\mathrm{E})$ and hydration $(\mathrm{H})$ at the volar forearm (VF), trochanter (TR) and the sacrum (SA), nutrition by a Mini Nutritional Assessment (MNA), total body water (TBW), lean body mass (LBM), \% body fat (\%F) by bioimpedance and routine laboratory parameters (hemoglobin, hematocrit, leukocytes, C-reactive protein, serum proteins and creatinine). Results: Mean body mass index (27 \pm 4.2$)$, MNA $(22.5 \pm 2.9)$, Braden score $(20 \pm 2.5), \mathrm{E}(68.5 \pm 6.0 \%)$ and $\mathrm{H}$ $(38.3 \pm 6.7)$ at any site and laboratory parameters did not differ by sex. Men had more TBW (+12 \pm 1.5 liters), LBM (+9 $\pm 2 \mathrm{~kg})$, less $\% \mathrm{~F}(-8.8 \pm 2.1 \%)$, increased H-TR $(+7.11 \pm 2.8)$
\end{abstract}

and H-SA $(+5.68 \pm 2.5)$. Overall E-VF correlated significantly with E-TR $\left(r^{2}=0.40, p<0.0001\right)$ and E-SA $\left(r^{2}=0.40, p<0.0001\right)$. In contrast, skin hydration was not correlated. Conclusion: Results of forearm elasticity experiments can be used as a model for other body sites at risk for the development of pressure ulcers.

Copyright $\odot 2012$ S. Karger AG, Basel

\section{Introduction}

The skin is a visible indicator of aging in humans. With aging, environmental, structural and functional alterations increase skin vulnerability and decrease barrier function. From a biomechanical view, aged skin is characterized by a decrease in elasticity (resilience) and thickness due to elastin fiber degradation and the disorganization and fragmentation of collagen. Reductions of epidermal cell number, water and lipids respectively in the stratum corneum are characteristics for xerosis and wrinkles. Functional decline, delirium or immobility are risk factors predisposing to decubitus ulcers (DU) over bony prominences at body sites exposed to high pressure (e.g. pelvic, trochanteric area, lower limbs). 
A decubitus is an area of localized skin damage and underlying tissue caused by prolonged tissue compression, friction and shear forces or a combination of these factors (www.epuap.org). Currently two types have been proposed: superficial ulcers primarily caused by moisture and friction/shear and deep tissue injury ulcers beginning in the subcutaneous fat or muscle induced by sustained loads. Since all DU are associated with high morbidity and mortality and lead to enormous costs, the early identification of patients at risk is an important clinical task. To date, several clinical scores are available to assess decubitus risk in bedridden patients.

Biophysical characteristics of skin have been associated with DU in several studies. Shear forces induce ischemia while skin hydration increases friction. We recently reported that forearm skin friction against selected medical textiles (cotton-polyester, viscose) did not differ between young and aged persons and that skin hydration increased while skin elasticity/resilience decreased with age. In the current study in old patients with a low decubitus risk in an acute-subacute disease stage, we examined the question if skin viscoelasticity and skin hydration at different areas prone to decubitus would correlate with similar measurements performed at the forearm.

\section{Methods}

\section{Study Design}

A total of 32 aged persons (18 women, 14 men) were recruited from our institution, an 88-bed acute geriatric hospital (table 1). Within this institution the average hospital stay of patients is 27 days. Reasons for admission were falls and/or fall related injury, acute surgery and acute osteoporotic fractures. Most frequent additional diagnoses were: post fall anxiety, cardiac failure, stable coronary heart disease and type 2 diabetes.

Patients were eligible based upon a detailed history and complete physical examination. Exclusion criteria were poor cognitive status, lack of consciousness, presence of skin lesions in the study areas, end-stage medical disease or cancer and any conditions or medication affecting skin thickness (e.g. corticosteroids), history of skin disease, allergies, tobacco use ( $>15$ cigarettes/day) and tattoos. All participants gave written informed consent. The ethics committee of the Kanton St. Gallen approved the study protocol (EKSG 07/019/1B).

\section{Skin Analysis}

All analyses were performed following removal of excessive hair and acclimatization and resting period of $15 \mathrm{~min}$ at $22 \pm 2{ }^{\circ} \mathrm{C}$ and $50 \pm 5 \%$ relative humidity. The participants refrained from applying cosmetics on the test site for 2 days before the measurements, and from washing their forearm with soap $6 \mathrm{~h}$ before skin testing. Skin experiments were carried out in the afternoon (1-5 p.m.). The viscoelastic properties were characterized using a suc-
Table 1. Baseline characteristics of study population

\begin{tabular}{lccl}
\hline & $\begin{array}{l}\text { Women } \\
(\mathrm{n}=18)\end{array}$ & $\begin{array}{l}\text { Men } \\
(\mathrm{n}=14)\end{array}$ & $\mathrm{p}$ \\
\hline Age & $82.4 \pm 4.6$ & $81.3 \pm 7.5$ & 0.6 \\
Body mass index & $26.6 \pm 4.5$ & $27.5 \pm 3.9$ & 0.6 \\
Mini Nutrition Assessment & $22.1 \pm 2.6$ & $22.3 \pm 0.3$ & 0.9 \\
Hematocrit & $0.4 \pm 0.0$ & $0.4 \pm 0.1$ & 0.8 \\
Hemoglobin, g/l & $122.1 \pm 17.6$ & $121.4 \pm 16.3$ & 0.9 \\
Leukocyte count, $\times 10^{9} / 1$ & $6.2 \pm 1.7$ & $6.2 \pm 1.3$ & 0.6 \\
Total proteins, g/l & $62.5 \pm 4.6$ & $60.6 \pm 8.4$ & 0.4 \\
Creatinine, $\mu$ mol/l & $83.8 \pm 39.1$ & $102.9 \pm 27.6$ & 0.1 \\
C-reactive protein, mg/l & $7.8 \pm 10.3$ & $5.3 \pm 4.0$ & 0.5 \\
Total body water, liters* & $28.6 \pm 3.1$ & $40.7 \pm 5.5$ & 0.0001 \\
Lean body mass, kg* & $38.9 \pm 4.2$ & $55.8 \pm 7.9$ & 0.0001 \\
Fat mass, kg* & $24.2 \pm 6.7$ & $23.6 \pm 9.1$ & 0.9 \\
\% Fat* & $37.9 \pm 5.0$ & $28.9 \pm 6.1$ & 0.0002 \\
Nutritional index & $75.6 \pm 13.2$ & $78.8 \pm 13.5$ & 0.5 \\
Braden score, points & $20.7 \pm 2.5$ & $20.0 \pm 2.6$ & 0.5 \\
\hline
\end{tabular}

$p$ values indicate differences between women and men.

* Bioimpedance analysis.

tion device (Cutometer ${ }^{\circledR}$ MPA 580; Courage + Khazaka, Cologne, Germany). Cutometer measurements were performed on 6 different locations of the inner forearm and on 5 locations over the sacrum and the trochanter. Viscoelastic skin resilience (overall elasticity $\mathrm{R} 2=\mathrm{Ua} / \mathrm{Uf})$, including creep and creep recovery, the pure elasticity $(\mathrm{R} 5=\mathrm{Ur} / \mathrm{Ue})$, skin viscosity $\mathrm{R} 6(\mathrm{Uv} / \mathrm{Ue})$, as well as the biological elasticity $(\mathrm{R} 7=\mathrm{Ur} / \mathrm{Uf})$ were determined from the skin deformation curve arising from a suction-relaxation cycle. Skin hydration was assessed at about 20-25 locations on the inner forearm, sacrum and trochanteric region using a Corneometer ${ }^{\circledR}$ probe (CM 825; Courage + Khazaka). Corneometer measurements are given in arbitrary units. CM values $<30$ characterize very dry, between 30 and 40 dry, and $>40$ normally moist skin.

\section{Assessment of Body Composition}

Body mass index was calculated as weight $(\mathrm{kg}) /$ height $\left(\mathrm{m}^{2}\right)$. One single experienced investigator performed all skin experiments, body composition was assessed by bioimpedance (BodyExplorer 2006; Juwell Medical, Gauting, Germany). In each patient we assessed total body mass (kg), \% body fat mass (\%F), lean body mass $(\mathrm{kg})$ and total body water (l).

\section{Clinical Assessment of Decubitus Risk}

The Braden score of all patients was assessed by a single-blinded nurse according to the recommendations by Braden and Bergstrom.

\section{Laboratory Analyses}

Blood samples were drawn in the fasting state. All tests were performed on automated analyzers (Synchron, Unicel DXC 800; Beckman Coulter, Inc., Fullerton, Calif., USA) using standardized clinical assays. 


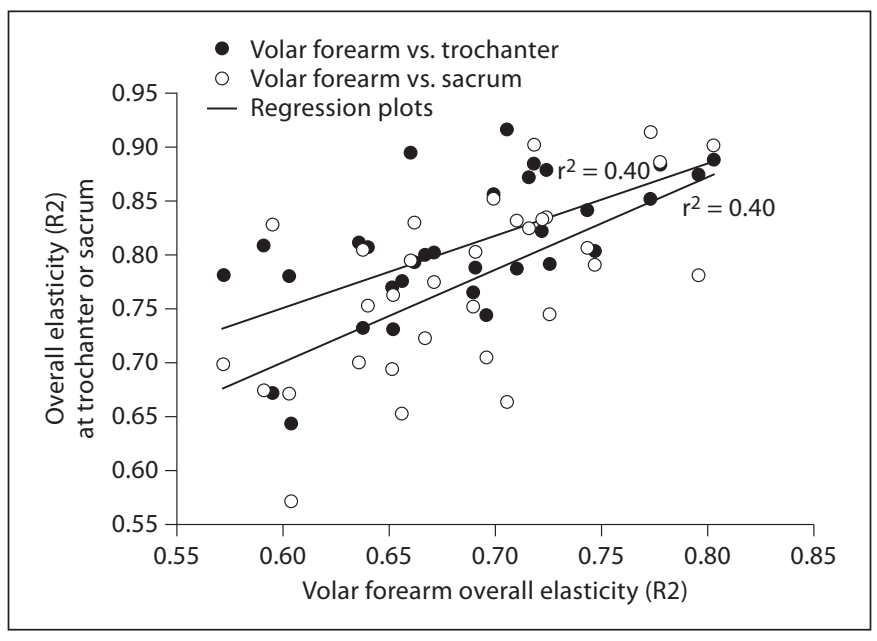

Fig. 1. Biophysical skin properties at different body sites.

Nutritional Assessment

Assessment of nutrition comprised the revised 2006 German version of the Mini Nutrition Assessment questionnaire which included measurements of circumferences of mid arm and calf respectively (www.mna-elderly.com).

\section{Statistics}

Analyses were done with Stata statistical software, release 9 (Stata Corp., College Station, Tex., USA). Summary statistics are described as means and standard deviations. Differences between categorical variables within each age group were assessed by $\chi^{2}$ tests and differences between continuous variables were calculated with Mann-Whitney tests or paired two-sided t tests where appropriate. Results of continuous data at different sites were compared by using simple regression analysis. A p value $<0.05$ was considered as significant.

\section{Results}

Study Population, Body Composition and Assessment of Decubitus Risk by Braden

We studied 32 geriatric patients with a mean age of $81.9 \pm 5.9$ years (table 1 ). Women and men did not differ by age, body mass index, nutrition score, laboratory parameters and Braden score. Men had more total body water $(+12.1 \pm 1.35$ liters, $\mathrm{p}<0.0001)$, a higher lean body mass $(+17.0 \pm 2.2 \mathrm{~kg}, \mathrm{p}<0.0001)$ and a lower relative fat mass $(-8.7 \pm 2.1 \%, \mathrm{p}<0.0001)$.

\section{Viscoelastic Skin Properties at Different Anatomical}

Regions

Skin elasticity in women and men differed according to sites. Overall elasticity (R2) in both women and men was highest at the trochanter (women $79 \%$, men $81 \%$ ), followed by the sacrum (women $77 \%$, men $76 \%$ ) and the forearm (women 67\%, men 69\%). Pure elasticity (R5), skin viscosity (R6) or biological elasticity (R7) at any of the sites - volar forearm, trochanter and sacrum - did not differ by sex (data not shown).

\section{Correlation of Biophysical Skin Properties at Different} Body Sites (fig. 1)

Overall forearm elasticity E-VF (R2) correlated significantly with corresponding (R2) elasticity measurements at the trochanter E-TR $\left(\mathrm{r}^{2}=0.40, \mathrm{p}<0.0001\right)$ and the sacrum E-SA $\left(r^{2}=0.40, p<0.0001\right)$. In contrast, we found no significant correlations of hydration measurements between volar forearm, trochanter and/or sacrum (data not shown).

\section{Discussion}

In this pilot study we found that skin resilience/elasticity at the volar forearm correlated with the trochanter and the sacrum. In addition, we demonstrated that men exhibited more total body water and also found a higher lean body mass but a lower relative fat mass confirming a previous report [1].

The forearm Cutometer results correlated significantly with those from the trochanter and sacrum. This finding is especially important for future research protocols using elasticity as a variable of interest because it allows estimating skin elasticity of other sites of the body. The reduced skin elasticity and turgor with aging (typically $10-35 \%$ lower than in the young) leads to increased skin wrinkling, skin tissue displacements, shear movements and distortions during friction contacts thereby increasing the risk for the development of superficial ulcers or abrasions. Our observations are consistent with previous findings on the effects of age, wetness and wrinkles on mechanical strains and stresses in the stratum corneum using finite element models. When comparing the volumetric skin tissue exposures to critical shear stresses, they found that the exposure of aged subcutis to potentially injurious shear stresses $(>70 \mathrm{kPa})$ is about $6-10$ times greater than in the young skin, for both pure tissue compression and combined compression-shear loading in a dry or wet environment [2]. This experiment provides data that age-related mechanical property changes are a risk factor for superficial DU.

In this study, we found slightly higher skin elasticity at the sacrum than the trochanter. According to earlier re- 
ports, the sacrum is the most frequent site of decubitus formation (26-36\% of all ulcers [3]). To which extent, however, differences in skin elasticity affect the development of DU at these anatomical sites is so far unknown. To our knowledge, the relationships between skin elasticity and DU formation at different anatomical regions have not been investigated in clinical trials. Furthermore, we found no significant correlation of hydration measurements at the volar forearm with trochanter and/or sacrum in our study population, the results indicating that skin moisture differs between exposed (arm) and non-exposed (trochanter) areas.

With aging, the water content of the skin decreases predominantly in the subcutis. As a consequence, the subcutis becomes less flexible and begins to fissure [4] so that application of humectants or moisturizing creams is necessary to protect aged skin [5]. We previously reported higher skin moisture in aged persons, the latter being attributed to the regular application of body care lotions containing urea leading to prolonged/persistent moisturizing effects [6]. Nevertheless, the results of the skin hydration measurement in our patients were on the lower limit of normal skin. The results of the small number of patients varied over a relatively large range (from 28 to 41). Physiologically, skin moisture is protective but wetness is problematic in areas prone to decubitus when wetness and consequently high friction may lead to skin damage. Elevated skin moisture levels (e.g. due to incontinence, high ambient temperature or hyperhidrosis) macerate the skin, which can result in the loss of mechanical strength, greater susceptibility to skin injury or higher risk of infection.

In this study, we used the Braden scale to clinically assess DU risk because it considers extrinsic (friction, moisture) and intrinsic risk factors. This scoring system is limited because additional patient-related information such as body temperature, body weight, polymorbidity, sex, age are usually not considered with the score. In addition, nurses must be very well trained to be able to institute appropriate interventions. Therefore it would be important to combine clinical and biophysical measurements to improve the predictive value [7]. The small sample size and the low decubitus risk of our study population did not allow such an extension of our research question. Nevertheless, other groups have recently approached this problem in younger patients using different technologies. One recent study reported in 105 intensive care patients that non-invasive skin interface pressure measurement at different sites provided a better DU assessment than the Braden scale [8]. Another group evaluated ultra- sound studies and hypothesized a strong association of skin thickness and DU risk [2].

Our study has several limitations. First our patients had a high functional level on admission and were mobilized as early as possible. Therefore, a similar study should be performed with a heterogeneous population including persons at high risk such as acute care, non-geriatric hospital, nursing-home residents. In addition, the study was performed during a short period of 5 months between May and September. Thus we cannot rule out that variations in climate during this period might have influenced our bedside measurements of skin hydration. The application of skin care products was discontinued 2 days before our measurements and long-term topical effects may have influenced our results. Finally, we did not routinely measure serum zinc levels to estimate of the nutritional status.

In summary, the results of our pilot study demonstrate that body composition differs between sex even in high age and that skin elasticity seems to be comparable while skin hydration varies between different body sites. Given the complexity of the structure of human skin on the one hand and discrepancy between risk assessment and decubitus prevalence on the other hand, future studies should focus on combined clinical measures (e.g. biochemical markers for deep tissue injury [9]). In addition, gero-technological innovations such as pressure-reducing spacer fabrics, medical textiles with low-friction properties [10] and drug delivery function, intelligent bed systems inducing position changes of bedridden persons might be future options for the prevention of a decubitus.

\section{Acknowledgements}

The authors wish to thank all the volunteers for their participation and patience. We also thank V. Strässle and A. Lenz (both Empa, St. Gallen, Switzerland) for support during the measuring setup, the team of the Geriatrische Klinik St. Gallen for the considerable help during the study, and two anonymous reviewers for their constructive critique and helpful comments for improving the manuscript.

\section{Funding}

Dr. Münzer was supported by a grant from the Robert Bosch Foundation (Stuttgart, Germany). This research was funded by the Swiss Commission for Technology and Innovation (KTI/ CTI), CTI project No. 7862.2, as well as industrial partners. 


\section{References}

$>1$ Dao H Jr, Kazin RA: Gender differences in skin: a review of the literature. Gend Med 2007;4:308-328.

-2 Sopher R, Gefen A: Effects of skin wrinkles, age and wetness on mechanical loads in the stratum corneum as related to skin lesions. Med Biol Eng Comput 2011;49:97-105.

$\checkmark 3$ Whittington KT, Briones R: National prevalence and incidence study: 6-year sequential acute care data. Adv Skin Wound Care 2004; 17:490-494.

4 McCallion R, Li Wan Po A: Dry and photoaged skin: manifestations and management. J Clin Pharm Ther 1993;18:15-32.
5 Reddy M, Gill SS, Rochon PA: Preventing pressure ulcers: a systematic review. JAMA 2006;296:974-984.

6 Gerhardt LC, Lenz A, Spencer ND, Münzer T, Derler S: Skin-textile friction and skin elasticity in young and aged persons. Skin Res Technol 2009;15:288-298.

7 Anthony D, Papanikolaou P, Parboteeah S, Saleh M: Do risk assessment scales for pressure ulcers work? J Tissue Viability 2010;19: 132-136.
8 Suriadi-Sanada H, Sugama J, Thigpen B, Kitagawa A, Kinosita S, Murayama S: A new instrument for predicting pressure ulcer risk in an intensive care unit. J Tissue Viability 2006;16:21-26.

9 Minematsu T, Nakagami G, Sari Y, Akase T, Sugama J, Nagase T, Sanada H: Candidate biomarkers for deep tissue damage from molecular biological and biochemical aspects. J Tissue Viability 2010;19:77-83.

10 Derler S, Rao A, Ballistreri P, Huber R, Scheel-Sailer A, Rossi RM: Medical textiles with low friction for decubitus prevention. Tribol Int 2012;46:208-214. 